\title{
BERRY-ESSÉEN BOUND FOR THE PARAMETER ESTIMATION OF FRACTIONAL ORNSTEIN-UHLENBECK PROCESSES WITH THE HURST PARAMETER $H \in\left(0, \frac{1}{2}\right)$
}

\author{
YONG CHEN AND YING LI
}

\begin{abstract}
For an Ornstein-Uhlenbeck process driven by a fractional Brownian motion with Hurst parameter $H \in\left(0, \frac{1}{2}\right)$, one shows the Berry-Esséen bound of the least squares estimator of the drift parameter. Thus, a problem left in Chen, Kuang, and Li 2018 is solved, where the Berry-Esséen bound of the least squares estimator is proved for $H \in\left[\frac{1}{2}, \frac{3}{4}\right]$. A new ingredient is a corollary of the inner product's representation of the Hilbert space associated with the fractional Brownian motion given by Jolis 2007. An approach based on Malliavin calculus given by Kim and Park 2017b is used. Several computations are cited from Hu, Nualart, and Zhou 2019.

Keywords: Berry-Esséen bound; Fourth Moment theorems; fractional Ornstein-Uhlenbeck process; Malliavin calculus.

MSC 2000: 60H07; 60F 25; 62M09.
\end{abstract}

\section{INTRODUCTION}

The statistical aspects of the following 1-dimensional Ornstein-Uhlenbeck process has been intensively studied by some authors recently.

$$
\mathrm{d} X_{t}=-\theta X_{t} \mathrm{~d} t+\mathrm{d} B_{t}^{H}, \quad X_{0}=0, \quad 0 \leq t \leq T,
$$

where $B_{t}^{H}$ be a 1-dimensional fractional Brownian motion with Hurst parameter $H \in(0,1)$. Suppose that $H$ is fixed and known, then there are several types of estimators to the drift coefficient.

Based on the continuous observation, the following maximum likelihood estimator is proposed:

$$
\hat{\theta}_{M L E}=-\left\{\int_{0}^{T} Q^{2}(s) \mathrm{d} w_{s}^{H}\right\}^{-1} \int_{0}^{T} Q(s) \mathrm{d} Z(s),
$$

where

$$
\begin{array}{r}
Q(t)=\frac{\mathrm{d}}{\mathrm{d} w_{t}^{H}} \int_{0}^{t} k_{H}(t, s) X_{s} \mathrm{~d} s, \quad Z(t)=\int_{0}^{t} k_{H}(t, s) \mathrm{d} X_{s}, \\
k_{H}(t, s)=\kappa_{H}^{-1} s^{\frac{1}{2}-H}(t-s)^{\frac{1}{2}-H}, \quad w_{t}^{H}=\lambda_{H}^{-1} t^{2-2 H}
\end{array}
$$

with constants $\kappa_{H}, \lambda_{H}$ depending on $H$. Please refer to Kleptsyna and Le Breton 2002 and Tudor and Viens 2007, where the almost sure convergence of both the MLE and a version of the MLE using discrete observations for all $H \in(0,1)$ is shown. Later on, the central limit theorem of $\hat{\theta}_{M L E}$ is shown in Bercu, Coutin, and Savy 2011 and Brouste and Kleptsyna 2010. 
The least squares estimator of the drift coefficient is given by a ratio of two Gaussian functionals ( $\mathrm{Hu}$ and Nualart 2010):

$$
\hat{\theta}_{T}=-\frac{\int_{0}^{T} X_{t} \mathrm{~d} X_{t}}{\int_{0}^{T} X_{t}^{2} \mathrm{~d} t}=\theta-\frac{\int_{0}^{T} X_{t} \mathrm{~d} B_{t}^{H}}{\int_{0}^{T} X_{t}^{2} \mathrm{~d} t}
$$

where $\mathrm{d} B_{t}^{H}$ denotes the divergence integral or the extended divergence integral (see Cheridito and Nualart 2005). In case of $H \in\left(0, \frac{3}{4}\right]$, the strong consistency and asymptotic normality of the estimator $\hat{\theta}_{T}$ are shown in Hu and Nualart 2010 and Hu, Nualart and Zhou 2019. It is worth noting that several crucial computations in this paper come from that given in $\mathrm{Hu}$, Nualart and Zhou 2019.

It is well known that $\hat{\theta}_{T}$ cannot be computed from the path of $X$ since the translation between divergence and Young integrals relies on the parameter $\theta$ that is being estimated. This makes many authors study other more practical and difficult parameter estimate based on discrete observations (e.g. Barboza and Viens 2017; Es-Sebaiy and Viens 2016; Sottinen and Viitasaari 2018). For example, in Es-Sebaiy 2013, a discrete time least squares estimator

$$
\hat{\theta}_{n}:=-\frac{\sum_{i=1}^{n} X_{t_{i-1}}\left(X_{t_{i}}-X_{t_{i-1}}\right)}{\Delta_{n} \sum_{i=1}^{n} X_{t_{i}}^{2}},
$$

where $t_{i}=i \Delta_{n}$, is proposed and an upper Berry-Esséen-type bound in the Kolmogorov distance for $\hat{\theta}_{n}$ is shown when $\Delta_{n} \rightarrow 0$ and $n \rightarrow \infty$. Moreover, the so-called "polynomial variation" estimator is proposed and an upper Berry-Esséen-type bound in the Wasserstein distance is shown in Onsy, Es-Sebaiy, and Viens 2017. It is also found out that to discretize the continuous-time estimator will lost the estimator's interpretation as a least square optimizer (Onsy, Es-Sebaiy, and Viens 2017).

But it is still meaningful to study the property of $\hat{\theta}_{T}$ because it is a first step to understand the problem of parameter estimate for the 1-dimensional fractional Ornstein-Uhlenbeck process (1.1) such as its Berry-Esséen behavior. Recently, by using an approach based on Malliavin calculus given by Kim and Park 2017b, it is shown in Chen, Kuang, and Li 2018 that as $T \rightarrow \infty$, when $H \in\left[\frac{1}{2}, \frac{3}{4}\right)$, the Berry-Esséen bound of $\sqrt{T}\left(\hat{\theta}_{T}-\theta\right)$ in the Kolmogorov distance is $\frac{1}{T^{\beta}}$, where $\beta=\frac{1}{2}, \frac{3}{8}-, 3-4 H$ for $H \in\left[\frac{1}{2}, \frac{5}{8}\right), H=\frac{5}{8}, H \in\left(\frac{5}{8}, \frac{3}{4}\right)$ respectively; when $H=\frac{3}{4}$, the Berry-Esséen bound of $\sqrt{\frac{T}{\log T}}\left(\hat{\theta}_{T}-\theta\right)$ in the Kolmogorov distance is $\frac{1}{\log T}$. In fact, when $H=\frac{1}{2}$, the Berry-Esséen bound of $\sqrt{T}\left(\hat{\theta}_{T}-\theta\right)$ in the Kolmogorov distance is well known, please refer to Bishwal 2000, 2008, and the references therein.

Since it involves much more complicated method to calculate the inner product of the Hilbert space associated to the fractional Brownian motion in the case of $H \in\left(0, \frac{1}{2}\right)$, the Berry-Esséen bound of $\sqrt{T}\left(\hat{\theta}_{T}-\theta\right)$ is still unknown for $H \in\left(0, \frac{1}{2}\right)$. In this paper, we will give an affirmative answer to this question. The main result of the present paper is as follows.

Theorem 1.1. Let $Z$ be a standard Gaussian random variable. When $H \in\left(0, \frac{1}{2}\right)$, there exists a constant $C_{\theta, H}$ such that when $T$ is large enough,

$$
\sup _{z \in \mathbb{R}}\left|P\left(\sqrt{\frac{T}{\theta \sigma_{H}^{2}}}\left(\hat{\theta}_{T}-\theta\right) \leq z\right)-P(Z \leq z)\right| \leq \frac{C_{\theta, H}}{T^{(1-2 H) \wedge \frac{1}{2}}} ;
$$


where $\sigma_{H}^{2}$ is given in Hu, Nualart, and Zhou 2019 as follows:

$$
\sigma_{H}^{2}=(4 H-1)+\frac{2 \Gamma(2-4 H) \Gamma(4 H)}{\Gamma(2 H) \Gamma(1-2 H)} .
$$

Proof of Theorem 1.1 will be given in Section 3. The main idea to show Theorem 1.1 will be given in Section 2.

Theorem 1.1 implies that when $H \in\left(0, \frac{1}{4}\right]$, the Berry-Esséen bound is $\frac{c}{\sqrt{T}}$. When $H \in\left(\frac{1}{4}, \frac{1}{2}\right)$, the Berry-Esséen bound is $\frac{c}{T^{1-2 H}}$. It is known that when $H=\frac{1}{2}$, the optimal Berry-Esséen bound is $\frac{c}{\sqrt{T}}$ (Kim and Park 2017a, 2017b). Thus, it is reasonable to conjecture that when $H \in\left(\frac{1}{4}, \frac{1}{2}\right)$, a better bound should be $\frac{c}{\sqrt{T}}$. This improving topic will be investigated in other works. In the remaining part of this paper, $c$ will be a generic positive constant whose values may differ from line to line.

\section{Preliminary}

The fractional Brownian motion (fBm) $B^{H}=\left\{B_{t}^{H}, t \in[0, T]\right\}$ with Hurst parameter $H \in$ $(0,1)$ is a continuous centered Gaussian process, defined on a complete probability space $(\Omega, \mathcal{F}, P)$, with covariance function given by

$$
\mathbb{E}\left(B_{t}^{H} B_{s}^{H}\right)=R_{H}(t, s)=\frac{1}{2}\left(|t|^{2 H}+|s|^{2 H}-|t-s|^{2 H}\right) .
$$

Let $\mathcal{E}$ denote the space of all real valued step functions on $[0, T]$. The Hilbert space $\mathfrak{H}$ is defined as the closure of $\mathcal{E}$ endowed with the inner product

$$
\left\langle\mathbf{1}_{[a, b)}, \mathbf{1}_{[c, d)}\right\rangle_{\mathfrak{H}}=\mathbb{E}\left(\left(B_{b}^{H}-B_{a}^{H}\right)\left(B_{d}^{H}-B_{c}^{H}\right)\right) .
$$

In the case $H \leq \frac{1}{2}$, this space is a space of functions, and when $H>\frac{1}{2}$, this space contains distributions that are not given by functions, please refer to Jolis 2007, Pipiras and Taqqu 2000 and 2001.

The following proposition is an adaptation of Theorem 2.3 of Jolis 2007.

Proposition 2.1. Denote $\mathcal{V}_{[0, T]}$ the set of bounded variation functions on $[a, b]$. Then $\mathcal{V}_{[0, T]}$ is dense in $\mathfrak{H}$. Moreover, if $f, g \in \mathcal{V}_{[0, T]}$, one has that

$$
\begin{aligned}
\langle f, g\rangle_{\mathfrak{H}} & =\int_{[0, T]^{2}} R_{H}(t, s) \nu_{f}(\mathrm{~d} t) \nu_{g}(\mathrm{~d} s), \\
& =-\int_{[0, T]^{2}} f(t) \frac{\partial R_{H}(t, s)}{\partial t} \mathrm{~d} t \nu_{g}(\mathrm{~d} s) .
\end{aligned}
$$

where $\nu_{g}$ is the restriction to $([0, T], \mathcal{B}([0, T]))$ of the Lebesgue-Stieljes signed measure associated with $g^{0}$ defined as

$$
g^{0}(x)= \begin{cases}g(x), & \text { if } x \in[0, T] . \\ 0, & \text { otherwise. }\end{cases}
$$

Proof. The first claim and the identity (2.1) are cited from Theorem 2.3 of Jolis 2007. The identity (2.2) can be implied from the formula of integrations by parts. In fact, for the step 
functions on $[0, T]$ of the form

$$
f=\sum_{j=0}^{N-1} f_{j} \mathbf{1}_{\left[t_{j}, t_{j+1}\right)},
$$

where $\left\{0=t_{0}<t_{1}<\cdots<t_{N}=T\right\}$ is a partition of $[0, T]$ and $f_{j} \in \mathbb{R}$. The corresponding signed measure is

$$
\nu_{f}=\sum_{j=1}^{N-1}\left(f_{j}-f_{j-1}\right) \delta_{t_{j}}+f(0+) \delta_{0}-f(T-) \delta_{T} .
$$

It is clear that the following formula of integrations by parts hold: for any $s \in[0, T]$,

$$
-\int_{[0, T]} f(t) \frac{\partial R_{H}(t, s)}{\partial t} \mathrm{~d} t=\int_{[0, T]} R_{H}(t, s) \nu_{f}(\mathrm{~d} t) .
$$

Next, given $f$ a right continuous monotone non-decreasing function on $[0, T]$ and a sequence partitions $\pi_{n}=\left\{0=t_{0}^{n}<t_{1}^{n}<\cdots<t_{k_{n}}^{n}=T\right\}$ such that $\pi_{n} \subset \pi_{n+1}$ and $\left|\pi_{n}\right| \rightarrow 0$ as $n \rightarrow \infty$, consider

$$
f_{n}=\sum_{j=0}^{k_{n}-1} f\left(t_{j}^{n}\right) \mathbf{1}_{\left[t_{j}^{n}, t_{j+1}^{n}\right)} .
$$

Hence, the sequence of signed measures $\nu_{f_{n}}$ converges weakly to $\nu_{f}$. Taking limit on both sides of (2.3), one has that it is still valid for right continuous monotone non-decreasing functions on $[0, T]$. Finally, it is well known that every function of bounded variation is the difference of two monotone non-decreasing function and that the value of $f$ at its points of discontinuity are irrelevant for the purposes of determining the Lebesgue-Stieltjes measure $\nu_{f}$ (Tao 2011). One has that (2.3) is valid for any $f \in \mathcal{V}_{[0, T]}$ and hence the identity (2.2) holds.

Especially, taking $g=h \cdot \mathbf{1}_{[a, b]}(\cdot)$ with $h$ a continuously differentiable function in (2.2), one has a more explicit inner product presentation using the distributional derivative.

Corollary 2.2. Denote by $\delta_{a}(\cdot)$ the Dirac delta function centered at a point a. Let $g=h \cdot \mathbf{1}_{[0, T]}(\cdot)$ with $h$ a continuously differentiable function. Then one has

$$
\begin{aligned}
\|g\|_{\mathfrak{H}}^{2}= & -\int_{[0, T]^{2}} h(t) h^{\prime}(s) \mathbf{1}_{[0, T]}(s) \frac{\partial R_{H}(t, s)}{\partial t} \mathrm{~d} t \mathrm{~d} s \\
& +\int_{[0, T]^{2}} h(t) h(s) \frac{\partial R_{H}(t, s)}{\partial t}\left[\delta_{T}(s)-\delta_{0}(s)\right] \mathrm{d} t \mathrm{~d} s .
\end{aligned}
$$

Proof. The Heaviside step function $\mathrm{H}(x)$ is defined as

$$
\mathrm{H}(x)= \begin{cases}1, & \text { if } x>0 \\ 0, & \text { if } x<0\end{cases}
$$

The distributional derivative of the Heaviside step function is the Dirac delta function:

$$
\frac{\mathrm{dH}(x)}{\mathrm{d} x}=\delta_{0}(x) \text {. }
$$


Hence, one has that

$$
\frac{\mathrm{d}}{\mathrm{d} x} \mathbf{1}_{[0, T]}(x)=\frac{\mathrm{d}}{\mathrm{d} x}[\mathrm{H}(x)-\mathrm{H}(x-T)]=\delta_{0}(x)-\delta_{T}(x),
$$

which implies that

$$
\nu_{g}(\mathrm{~d} x)=\left[h^{\prime}(x) \mathbf{1}_{[a, b]}(x)+h(x)\left(\delta_{0}(x)-\delta_{T}(x)\right)\right] \mathrm{d} x .
$$

Substituting (2.6) into (2.2), one has the desired inner product presentation (2.4).

Remark 2.3. If $f, g \in \mathfrak{H}$ and $g$ is a continuously differentiable function with compact support, it is proved in Hu, Jolis, and Tindel 2013 and Hu, Nualart and Zhou 2019 that

$$
\langle f, g\rangle_{\mathfrak{H}}=-\int_{[0, T]^{2}} f(t) g^{\prime}(s) \frac{\partial R_{H}(t, s)}{\partial t} \mathrm{~d} t \mathrm{~d} s .
$$

The corollary implies that if $g^{\prime}$ is interpreted as the distributional derivative, then the identity (2.7) maybe still holds for the functions such as $g=h \cdot \mathbf{1}_{[a, b]}(\cdot)$ with $h$ a continuously differentiable function. But one will not attempt to prove it here since the theory of fractional order Sobolev spaces is involved.

It is well known that when $H \in\left(\frac{1}{2}, 1\right)$, for any $f, g \in L^{\frac{1}{H}}([0, T])$, if one extends $f$ and $g$ to be zero on $\mathbb{R} \cap[0, T]^{c}$, then $f, g \in \mathfrak{H}$ and (2.7) is equal to a simple identity

$$
\langle f, g\rangle_{\mathfrak{H}}=H(2 H-1) \int_{[0, T]^{2}} f(u) g(v)|u-v|^{2 H-2} \mathrm{~d} u \mathrm{~d} v .
$$

As one points out before, it is the difference between (2.4) and (2.8) that leads to the case of $H \in\left(0, \frac{1}{2}\right)$ much more complicated than the case of $H \in\left[\frac{1}{2}, \frac{3}{4}\right]$.

A Gaussian isonormal process associated with $\mathfrak{H}$ is given by Wiener integrals with respect to a $\mathrm{fBm}$ for any deterministic kernel $f \in \mathfrak{H}$ :

$$
B^{H}(f)=\int_{0}^{\infty} f(s) \mathrm{d} B_{s}^{H}
$$

Let $H_{n}$ be the $n$-th Hermite polynomial. The closed linear subspace $\mathfrak{H}_{n}$ of $L^{2}(\Omega)$ generated by $\left\{H_{n}\left(B^{H}(f)\right): f \in \mathfrak{H},\|f\|_{\mathfrak{H}}=1\right\}$ is called the $n$-th Wiener-Ito chaos. The linear isometric mapping $I_{n}: \mathfrak{H}^{\odot n} \rightarrow \mathfrak{H}_{n}$ given by $I_{n}\left(h^{\otimes n}\right)=H_{n}\left(B^{H}(f)\right)$ is called the $n$-th multiple Wiener-Ito integral. For any $f \in \mathfrak{H}^{\otimes n}$, define $I_{n}(f)=I_{n}(\tilde{f})$ where $\tilde{f}$ is the symmetrization of $f$.

Given $f \in \mathfrak{H}^{\odot p}$ and $g \in \mathfrak{H}^{\odot q}$ and $r=1, \cdots, p \wedge q, r$-th contraction between $f$ and $g$ is the element of $\mathfrak{H}^{\otimes(p+q-2 r)}$ defined by

$$
f \otimes_{r} g\left(t_{1}, \ldots, t_{p+q-2 r}\right)=\left\langle f\left(t_{1}, \ldots, t_{r}, \cdot\right), g\left(t_{r+1}, \ldots, t_{p+q-2 r}, \cdot\right)\right\rangle_{\mathfrak{H} \otimes r} .
$$

One will make use of the following estimate of the Kolmogrov distance between a nonlinear Gaussian functional and the standard normal (see Corollary 1 of Kim and Park 2017b).

Theorem 2.4 (Kim, Y. T., \& Park, H. S). Suppose that $\varphi_{T}(t, s)$ and $\psi_{T}(t, s)$ are two functions on $\mathfrak{H}^{\otimes 2}$. Let $b_{T}$ be a positive function of $T$ such that $I_{2}\left(\psi_{T}\right)+b_{T}>0$ a.s. If $\Psi_{i}(T) \rightarrow 0, i=1,2,3$ as $T \rightarrow \infty$, then there exists a constant $c$ such that for $T$ large enough,

$$
\sup _{z \in \mathbb{R}}\left|P\left(\frac{I_{2}\left(\varphi_{T}\right)}{I_{2}\left(\psi_{T}\right)+b_{T}} \leq z\right)-P(Z \leq z)\right| \leq c \times \max _{i=1,2,3} \Psi_{i}(T),
$$


where

$$
\begin{aligned}
& \Psi_{1}(T)=\frac{1}{b_{T}^{2}} \sqrt{\left[b_{T}^{2}-2\left\|\varphi_{T}\right\|_{\mathfrak{H}^{\otimes 2}}^{2}\right]^{2}+8\left\|\varphi_{T} \otimes_{1} \varphi_{T}\right\|_{\mathfrak{H}^{\otimes 2}}^{2}} \\
& \Psi_{2}(T)=\frac{2}{b_{T}^{2}} \sqrt{2\left\|\varphi_{T} \otimes_{1} \psi_{T}\right\|_{\mathfrak{H}^{\otimes 2}}^{2}+\left\langle\varphi_{T}, \psi_{T}\right\rangle_{\mathfrak{H}^{\otimes 2}}^{2}}, \\
& \Psi_{3}(T)=\frac{2}{b_{T}^{2}} \sqrt{\left\|\psi_{T}\right\|_{\mathfrak{H} \otimes 2}^{4}+2\left\|\psi_{T} \otimes_{1} \psi_{T}\right\|_{\mathfrak{H} \otimes 2}^{2}} .
\end{aligned}
$$

It follows from Eq.(1.2) and the product formula of multiple integrals that

$$
\sqrt{\frac{T}{\theta \sigma_{H}^{2}}}\left(\hat{\theta}_{T}-\theta\right)=\frac{I_{2}\left(f_{T}\right)}{I_{2}\left(g_{T}\right)+b_{T}},
$$

where

$$
\begin{aligned}
f_{T}(t, s) & =\frac{1}{2 \sqrt{\theta \sigma_{H}^{2} T}} e^{-\theta|t-s|} \mathbf{1}_{\{0 \leq s, t \leq T\}}, \\
g_{T}(t, s) & =\sqrt{\frac{\sigma_{H}^{2}}{\theta T}} f_{T}-\frac{1}{2 \theta T} h_{T}, \\
h_{T}(t, s) & =e^{-\theta(T-t)-\theta(T-s)} \mathbf{1}_{\{0 \leq s, t \leq T\}}, \\
b_{T} & =\frac{1}{T} \int_{0}^{T}\left\|e^{-\theta(t-\cdot)} 1_{[0, t]}(\cdot)\right\|_{\mathfrak{H}}^{2} \mathrm{~d} t .
\end{aligned}
$$

The reader can also refer to Eq.(17)-(19) of Kim and Park 2017a for details. By Theorem 2.4 and the identity (2.10), to obtain the Berry-Esséen bound of $\hat{\theta}_{T}$, one need to estimate the right hand side of (2.9) which are several integrals. This is the main idea of the present paper and the previous paper Chen, Kuang, and Li 2018.

\section{Proof of Theorem 1.1}

One divides the estimate of the right hand side of (2.9) into several lemmas. The following estimate is cited from the inequality (3.17) of Hu, Nualart, and Zhou 2019.

Lemma 3.1. When $H \in\left(0, \frac{1}{2}\right)$, there exists a constant $C_{\theta, H}$ such that

$$
\left\|f_{T} \otimes_{1} f_{T}\right\|_{\mathfrak{H}^{\otimes 2}} \leq \frac{C_{\theta, H}}{\sqrt{T}} .
$$

It is worth noting that to show the estimate (3.1), the Fourier transform is used to compute the inner product of the Hilbert space $\mathfrak{H}$ (Pipiras and Taqqu 2000):

$$
\langle f, g\rangle_{\mathfrak{H}}=\frac{\Gamma(2 H+1) \sin (\pi H)}{2 \pi} \int_{\mathbb{R}} \mathcal{F} f(\xi) \overline{\mathcal{F} g(\xi)}|\xi|^{1-2 H} \mathrm{~d} \xi .
$$

Although the estimate (3.1) is crucial to the present paper, one will not use this method to compute the inner product any more in this paper.

Lemma 3.2. When $H \in(0,1)$, the speed of convergence $b_{T} \rightarrow H \Gamma(2 H) \theta^{-2 H}$ as $T \rightarrow \infty$ is at least $\frac{1}{T}$. 
Proof. The symmetry and Corollary 2.2 imply that

$$
\begin{aligned}
b_{T} & =-\frac{1}{T} \int_{0}^{T} e^{-2 \theta t} \mathrm{~d} t \int_{\mathbb{R}^{2}} \frac{\partial}{\partial v}\left[e^{\theta(u+v)} \mathbf{1}_{[0, t]^{2}}(u, v)\right] \frac{\partial R_{H}(u, v)}{\partial u} \mathrm{~d} u \mathrm{~d} v \\
& =\frac{\theta H}{T} \int_{0}^{T} e^{-2 \theta t} \mathrm{~d} t \int_{[0, t]^{2}} e^{\theta(u+v)}\left(\operatorname{sgn}(u-v)|u-v|^{2 H-1}-|u|^{2 H-1}\right) \mathrm{d} u \mathrm{~d} v \\
& +\frac{H}{T} \int_{0}^{T} e^{-2 \theta t} \mathrm{~d} t \int_{\mathbb{R}^{2}} e^{\theta(u+v)} \mathbf{1}_{[0, t]}(u)\left(\delta_{0}(v)-\delta_{t}(v)\right)\left(\operatorname{sgn}(u-v)|u-v|^{2 H-1}-|u|^{2 H-1}\right) \mathrm{d} u \mathrm{~d} v \\
& =\frac{H}{T} \int_{0}^{T} e^{-2 \theta t} \mathrm{~d} t\left[\left(-\theta \int_{[0, t]^{2}} e^{\theta(u+v)} u^{2 H-1} \mathrm{~d} u \mathrm{~d} v+\int_{0}^{t} e^{\theta(u+t)} u^{2 H-1} \mathrm{~d} u\right)+\int_{0}^{t} e^{\theta(u+t)}(t-u)^{2 H-1} \mathrm{~d} u\right] \\
& =\frac{H}{T} \int_{0}^{T} e^{-2 \theta t} \mathrm{~d} t\left[\int_{0}^{t} e^{\theta u} u^{2 H-1} \mathrm{~d} u+\int_{0}^{t} e^{\theta(u+t)}(t-u)^{2 H-1} \mathrm{~d} u\right] \\
& :=B_{1}+B_{2},
\end{aligned}
$$

where $B_{1}, B_{2}$ and their convergence speeds are given respectively as follows. Integration by parts implies that there exists a constant $C_{\theta, H}$ such that

$$
\begin{aligned}
0<B_{1} & =\frac{H}{T} \int_{0}^{T} e^{-2 \theta t} \mathrm{~d} t \int_{0}^{t} e^{\theta u} u^{2 H-1} \mathrm{~d} u \\
& =\frac{H}{2 \theta T}\left[\frac{1}{e^{2 \theta T}} \int_{0}^{T} e^{\theta u} u^{2 H-1} \mathrm{~d} u+\int_{0}^{T} e^{-\theta t} t^{2 H-1} \mathrm{~d} t\right] \\
& \leq \frac{C_{\theta, H}}{T}
\end{aligned}
$$

Making change of variable $z=t-u$ and then integration by parts, one has that

$$
\begin{aligned}
B_{2}-H \Gamma(2 H) \theta^{-2 H} & =\frac{H}{T} \int_{0}^{T} e^{-\theta t} \mathrm{~d} t \int_{0}^{t} e^{\theta u}(t-u)^{2 H-1} \mathrm{~d} u-H \Gamma(2 H) \theta^{-2 H} \\
& =\frac{H}{T} \int_{0}^{T} \mathrm{~d} t \int_{0}^{t} e^{-\theta z} z^{2 H-1} \mathrm{~d} z-H \Gamma(2 H) \theta^{-2 H} \\
& =\frac{H}{T}\left[T \int_{0}^{T} e^{-\theta t} t^{2 H-1} \mathrm{~d} t-\int_{0}^{T} e^{-\theta t} t^{2 H} \mathrm{~d} t\right]-H \Gamma(2 H) \theta^{-2 H} \\
& =H \int_{T}^{\infty} e^{-\theta t} t^{2 H-1} \mathrm{~d} t-\frac{H}{T} \int_{0}^{T} e^{-\theta t} t^{2 H} \mathrm{~d} t
\end{aligned}
$$

Hence, there exists a constant $C_{\theta, H}^{\prime}$ such that

$$
\begin{aligned}
\left|B_{2}-H \Gamma(2 H) \theta^{-2 H}\right| & \leq H \int_{T}^{\infty} e^{-\theta t} t^{2 H-1} \mathrm{~d} t+\frac{H}{T} \int_{0}^{T} e^{-\theta t} t^{2 H} \mathrm{~d} t \\
& \leq \frac{C_{\theta, H}^{\prime}}{T}
\end{aligned}
$$

Combining the limits (3.3) and (3.4) with the equality (3.2), one has that the speed of convergence $b_{T} \rightarrow H \Gamma(2 H) \theta^{-2 H}$ is at least $\frac{1}{T}$.

Remark 3.3. In the case of $H \in\left[\frac{1}{2}, \frac{3}{4}\right)$, the same conclusion is shown in Chen, Kuang, and Li 2018. The proof in the present paper is suited to all $H \in(0,1)$. 
Lemma 3.4. Let $h_{T}$ be given as in (2.13) and $H \in\left(0, \frac{3}{4}\right)$. Then as $T \rightarrow \infty$,

$$
\frac{1}{\sqrt{T}} h_{T} \rightarrow 0, \quad \text { in } \quad \mathfrak{H}^{\otimes 2}
$$

Proof. Without loss of generality, we can assume that $\theta=1$. Denote $\vec{t}=\left(t_{1}, t_{2}\right), \vec{s}=\left(s_{1}, s_{2}\right)$. The identity (2.4) implies that

$$
\begin{aligned}
\frac{1}{T}\left\|h_{T}\right\|_{\mathfrak{H}^{\otimes 2}}^{2} & =\frac{1}{T e^{4 T}} \int_{\mathbb{R}^{4}} \frac{\partial^{2}}{\partial t_{1} \partial s_{2}}\left[e^{t_{1}+s_{1}+t_{2}+s_{2}} \mathbf{1}_{[0, T]^{4}}\left(t_{1}, s_{1}, t_{2}, s_{2}\right)\right] \frac{\partial R_{H}\left(t_{1}, t_{2}\right)}{\partial t_{2}} \frac{\partial R_{H}\left(s_{1}, s_{2}\right)}{\partial s_{1}} \mathrm{~d} \vec{t} \mathrm{~d} \vec{s} \\
& =\frac{1}{T e^{4 T}} \int_{\mathbb{R}^{4}} \mathbf{1}_{[0, T]^{2}}\left(s_{1}, t_{2}\right) e^{t_{1}+s_{1}+t_{2}+s_{2}} \frac{\partial R_{H}\left(t_{1}, t_{2}\right)}{\partial t_{2}} \frac{\partial R_{H}\left(s_{1}, s_{2}\right)}{\partial s_{1}} \\
& \times\left[\mathbf{1}_{[0, T]^{2}}\left(t_{1}, s_{2}\right)+\mathbf{1}_{[0, T]}\left(t_{1}\right)\left(\delta_{0}\left(s_{2}\right)-\delta_{T}\left(s_{2}\right)\right)\right. \\
& \left.+\mathbf{1}_{[0, T]}\left(s_{2}\right)\left(\delta_{0}\left(t_{1}\right)-\delta_{T}\left(t_{1}\right)\right)+\left(\delta_{0}\left(s_{2}\right)-\delta_{T}\left(s_{2}\right)\right)\left(\delta_{0}\left(t_{1}\right)-\delta_{T}\left(t_{1}\right)\right)\right] \mathrm{d} \vec{t} \mathrm{~d} \vec{s} \\
& :=I_{1}+I_{2}+I_{3}+I_{4} .
\end{aligned}
$$

By the symmetry and the L'Hospital's rule, one has that

$$
\begin{aligned}
\lim _{T \rightarrow \infty} I_{1} & =\lim _{T \rightarrow \infty} \frac{1}{T e^{4 T}} \int_{[0, T]^{4}} e^{t_{1}+s_{1}+t_{2}+s_{2}} \frac{\partial R_{H}\left(t_{1}, t_{2}\right)}{\partial t_{2}} \frac{\partial R_{H}\left(s_{1}, s_{2}\right)}{\partial s_{1}} \mathrm{~d} \vec{t} \mathrm{~d} \vec{s} \\
& =\lim _{T \rightarrow \infty} \frac{2}{T e^{4 T}}\left[\int_{0 \leq t_{2}, s_{1}, s_{2} \leq t_{1} \leq T}+\int_{0 \leq t_{1}, s_{1}, s_{2} \leq t_{2} \leq T} e^{t_{1}+s_{1}+t_{2}+s_{2}} \frac{\partial R_{H}\left(t_{1}, t_{2}\right)}{\partial t_{2}} \frac{\partial R_{H}\left(s_{1}, s_{2}\right)}{\partial s_{1}} \mathrm{~d} \vec{t} \mathrm{~d} \vec{s}\right. \\
& =\lim _{T \rightarrow \infty} \frac{2 H}{(1+4 T) e^{3 T}} \int_{[0, T]^{3}} e^{t+s_{1}+s_{2}}\left(T^{2 H-1}+t^{2 H-1}\right) \frac{\partial R_{H}\left(s_{1}, s_{2}\right)}{\partial s_{1}} \mathrm{~d} t \mathrm{~d} s_{1} \mathrm{~d} s_{2} \\
& =\lim _{T \rightarrow \infty} \frac{H^{2}}{2 T e^{3 T}} \int_{[0, T]^{3}} e^{t+s_{1}+s_{2}}\left(T^{2 H-1}+t^{2 H-1}\right) s_{1}^{2 H-1} \mathrm{~d} t \mathrm{~d} s_{1} \mathrm{~d} s_{2} \\
& =\lim _{T \rightarrow \infty} \frac{H^{2}}{2 T e^{2 T}} \int_{[0, T]^{2}} e^{t+s_{1}}\left(T^{2 H-1}+t^{2 H-1}\right) s_{1}^{2 H-1} \mathrm{~d} t \mathrm{~d} s_{1} \\
& =0 .
\end{aligned}
$$

In the same way, one has that as $T \rightarrow \infty$,

$$
I_{2}=I_{3}=-\frac{H}{T e^{3 T}} \int_{[0, T]^{3}} e^{s_{1}+t+s_{2}}\left(t^{2 H-1}+(T-t)^{2 H-1}\right) \frac{\partial R_{H}\left(s_{1}, s_{2}\right)}{\partial s_{1}} \mathrm{~d} t \mathrm{~d} s_{1} \mathrm{~d} s_{2} \rightarrow 0 .
$$

Finally, one has that as $T \rightarrow \infty$,

$$
\begin{aligned}
I_{4} & =\frac{H^{2}}{T e^{2 T}} \int_{[0, T]^{2}} e^{s+t}\left(t^{2 H-1}+(T-t)^{2 H-1}\right)\left(s^{2 H-1}+(T-s)^{2 H-1}\right) \mathrm{d} t \mathrm{~d} s \\
& =\frac{H^{2}}{T e^{2 T}}\left[\int_{0}^{T} e^{t}\left(t^{2 H-1}+(T-t)^{2 H-1}\right) \mathrm{d} t\right]^{2} \rightarrow 0 .
\end{aligned}
$$

Combining the above three limits with the equality (3.6), one has that $\frac{1}{T}\left\|h_{T}\right\|_{\mathfrak{H} \otimes 2}^{2} \rightarrow 0$ as $T \rightarrow \infty$.

Based on Lemma 3.4, one can obtain the following corollary whose proof is the same as Lemma 3.4 of Chen, Kuang, and Li 2018. 
Corollary 3.5. Let $g_{T}$ and $\sigma_{H}^{2}$ be given as in (2.12) and (1.4) respectively. Denote by $\delta_{H}=$ $H^{2} \Gamma^{2}(2 H) \sigma_{H}^{2}$. When $H \in\left(0, \frac{1}{2}\right)$, we have that as $T \rightarrow \infty$,

$$
\begin{aligned}
T\left\|g_{T}\right\|_{\mathfrak{H}^{\otimes 2}}^{2} & \rightarrow \frac{\delta_{H}}{2 \theta^{1+4 H}}, \quad T\left\langle f_{T}, g_{T}\right\rangle_{\mathfrak{H}^{\otimes 2}}^{2} \rightarrow \frac{\delta_{H}^{2}}{4 \theta^{1+8 H} \sigma_{H}^{2}}, \\
T\left\|f_{T} \otimes_{1} g_{T}\right\|_{\mathfrak{H}^{\otimes 2}}^{2} & \rightarrow 0, \quad T\left\|g_{T} \otimes_{1} g_{T}\right\|_{\mathfrak{H}^{\otimes 2}}^{2} \rightarrow 0 .
\end{aligned}
$$

Lemma 3.6. When $H \in\left(0, \frac{1}{2}\right)$, the speed of convergence $2\left\|f_{T}\right\|_{\mathfrak{H}^{\otimes 2}}^{2} \rightarrow\left[H \Gamma(2 H) \theta^{-2 H}\right]^{2}$ is at least $T^{2 H-1}$ as $T \rightarrow \infty$.

Proof. Without loss of generality, one can assume that $\theta=1$. One divide the proof into several steps.

Step 1. Similarly to obtain (3.6), one has that

$$
\begin{aligned}
2\left\|f_{T}\right\|_{\mathfrak{H}^{\otimes 2}}^{2} & =\frac{1}{2 T \sigma_{H}^{2}} \int_{\mathbb{R}^{4}} \frac{\partial^{2}}{\partial t_{1} \partial s_{2}}\left[e^{-\left|t_{1}-s_{1}\right|-\left|t_{2}-s_{2}\right|} \mathbf{1}_{[0, T)^{4}}\left(t_{1}, s_{1}, t_{2}, s_{2}\right)\right] \frac{\partial R_{H}\left(t_{1}, t_{2}\right)}{\partial t_{2}} \frac{\partial R_{H}\left(s_{1}, s_{2}\right)}{\partial s_{1}} \mathrm{~d} \vec{t} \mathrm{~d} \vec{s} \\
& =\frac{1}{2 T \sigma_{H}^{2}} \int_{\mathbb{R}^{4}} e^{-\left|t_{1}-s_{1}\right|-\left|t_{2}-s_{2}\right|} \mathbf{1}_{[0, T]^{2}}\left(s_{1}, t_{2}\right) \frac{\partial R_{H}\left(t_{1}, t_{2}\right)}{\partial t_{2}} \frac{\partial R_{H}\left(s_{1}, s_{2}\right)}{\partial s_{1}} \\
& \times\left[\mathbf{1}_{[0, T]^{2}}\left(t_{1}, s_{2}\right) \operatorname{sgn}\left(t_{1}-s_{1}\right) \operatorname{sgn}\left(s_{2}-t_{2}\right)-\mathbf{1}_{[0, T]}\left(t_{1}\right) \operatorname{sgn}\left(t_{1}-s_{1}\right)\left(\delta_{0}\left(s_{2}\right)-\delta_{T}\left(s_{2}\right)\right)\right. \\
& \left.-\mathbf{1}_{[0, T]}\left(s_{2}\right) \operatorname{sgn}\left(s_{2}-t_{2}\right)\left(\delta_{0}\left(t_{1}\right)-\delta_{T}\left(t_{1}\right)\right)+\left(\delta_{0}\left(s_{2}\right)-\delta_{T}\left(s_{2}\right)\right)\left(\delta_{0}\left(t_{1}\right)-\delta_{T}\left(t_{1}\right)\right)\right] \mathrm{d} \vec{t} \mathrm{~d} \vec{s} \\
& :=I_{1}(T)+I_{2}(T)+I_{3}(T)+I_{4}(T) .
\end{aligned}
$$

Step 2. Speed of convergence of $I_{1}(T) \rightarrow[H \Gamma(2 H)]^{2}$. It is proved in $\mathrm{Hu}$, Nualart, and Zhou 2019 that as $T \rightarrow \infty$,

$$
\begin{aligned}
I_{1}(T) & =\frac{1}{2 T \sigma_{H}^{2}} \int_{[0, T]^{4}} e^{-\left|t_{1}-s_{1}\right|-\left|t_{2}-s_{2}\right|} \frac{\partial R_{H}\left(t_{1}, t_{2}\right)}{\partial t_{2}} \frac{\partial R_{H}\left(s_{1}, s_{2}\right)}{\partial s_{1}} \operatorname{sgn}\left(t_{1}-s_{1}\right) \operatorname{sgn}\left(s_{2}-t_{2}\right) \mathrm{d} \vec{d} \mathrm{~d} \vec{s} \\
& \rightarrow[H \Gamma(2 H)]^{2} .
\end{aligned}
$$

Since $H \in\left(0, \frac{1}{2}\right)$, the symmetry and the L'Hospital's rule (Taylor 1952) imply that

$$
\begin{aligned}
& \limsup _{T \rightarrow \infty} T^{1-2 H} \frac{\sigma_{H}^{2}}{H^{2}}\left|I_{1}(T)-(H \Gamma(2 H))^{2}\right| \\
& =\limsup _{T \rightarrow \infty} \frac{\sigma_{H}^{2}}{T^{2 H}} \mid \frac{1}{2} \int_{[0, T]^{4}} e^{-\left|t_{1}-s_{1}\right|-\left|t_{2}-s_{2}\right|}\left(t_{2}^{2 H-1}-\operatorname{sgn}\left(t_{2}-t_{1}\right)\left|t_{2}-t_{1}\right|^{2 H-1}\right) \\
& \times\left(s_{1}^{2 H-1}-\operatorname{sgn}\left(s_{1}-s_{2}\right)\left|s_{1}-s_{2}\right|^{2 H-1}\right) \operatorname{sgn}\left(t_{1}-s_{1}\right) \operatorname{sgn}\left(s_{2}-t_{2}\right) \mathrm{d} \vec{t} \mathrm{~d} \vec{s}-\Gamma^{2}(2 H) \sigma_{H}^{2} T \mid \\
& \leq \frac{\sigma_{H}^{2}}{2 H} \limsup _{T \rightarrow \infty} T^{1-2 H}\left|I_{11}(T)+I_{12}(T)-\Gamma^{2}(2 H) \sigma_{H}^{2}\right|
\end{aligned}
$$

where

$$
\begin{aligned}
& I_{11}(T)= \\
& \int_{[0, T]^{3}} e^{t_{1}-T-\left|s-t_{2}\right|} \operatorname{sgn}\left(t_{2}-s\right)\left(T^{2 H-1}-(T-s)^{2 H-1}\right)\left(t_{2}^{2 H-1}-\operatorname{sgn}\left(t_{2}-t_{1}\right)\left|t_{2}-t_{1}\right|^{2 H-1}\right) \mathrm{d} s \mathrm{~d} \vec{t}
\end{aligned}
$$




$$
\begin{aligned}
& I_{12}(T)= \\
& \int_{[0, T]^{3}} e^{s_{1}-T-\left|s_{2}-t\right|} \operatorname{sgn}\left(s_{2}-t\right)\left(t^{2 H-1}+(T-t)^{2 H-1}\right)\left(s_{1}^{2 H-1}-\operatorname{sgn}\left(s_{1}-s_{2}\right)\left|s_{1}-s_{2}\right|^{2 H-1}\right) \mathrm{d} t \mathrm{~d} \vec{s},
\end{aligned}
$$

please refer to (6.30)-(6.31) of Hu, Nualart, and Zhou 2019.

Step 2.1. An expansion of $I_{11}$. After dividing the domain of integration $I_{11}$ into two domains according to $s>t_{2}$ or not and doing a change of variables as in (6.32) of Hu, Nualart, and Zhou 2019 , one has an expansion of $I_{11}$ as follows.

$$
\begin{aligned}
& I_{11}(T) \\
& =\int_{[0, T)^{3}, x \leq t} e^{-u-x}\left(T^{2 H-1}-t^{2 H-1}\right)\left((T-t+x)^{2 H-1}-\operatorname{sgn}(x+u-t)|x+u-t|^{2 H-1}\right) \mathrm{d} u \mathrm{~d} x \mathrm{~d} t \\
& -\int_{[0, T)^{3}, x \leq t} e^{-u-x}\left(T^{2 H-1}-(t-x)^{2 H-1}\right)\left((T-t)^{2 H-1}-\operatorname{sgn}(u-t)|u-t|^{2 H-1}\right) \mathrm{d} u \mathrm{~d} x \mathrm{~d} t \\
& =\int_{[0, T]^{2}} e^{-u-x} \sum_{i=1}^{4} \varphi_{i} \mathrm{~d} x \mathrm{~d} u,
\end{aligned}
$$

where

$$
\begin{aligned}
\varphi_{1}(x) & =\int_{x}^{T}\left((t-x)^{2 H-1}-T^{2 H-1}\right)\left((T-t)^{2 H-1}-(T-t+x)^{2 H-1}\right) \mathrm{d} t, \\
\varphi_{2}(x) & =\int_{x}^{T}\left((t-x)^{2 H-1}-t^{2 H-1}\right)(T-t+x)^{2 H-1} \mathrm{~d} t, \\
\varphi_{3}(x, u) & =T^{2 H-1} \int_{x}^{T}\left(\operatorname{sgn}(u-t)|u-t|^{2 H-1}-\operatorname{sgn}(x+u-t)|x+u-t|^{2 H-1}\right) \mathrm{d} t, \\
\varphi_{4}(x, u) & =\int_{x}^{T}\left(t^{2 H-1} \operatorname{sgn}(x+u-t)|x+u-t|^{2 H-1}-(t-x)^{2 H-1} \operatorname{sgn}(u-t)|u-t|^{2 H-1}\right) \mathrm{d} t .
\end{aligned}
$$

Step 2.2. Speed of convergence $\int_{[0, T]^{2}} e^{-u-x} \sum_{i=1}^{3}\left|\varphi_{i}\right| \mathrm{d} x \mathrm{~d} u \rightarrow 0$. For any fixed $\epsilon \in\left(0, \frac{1}{4}\right)$, denote $\mathcal{I}_{1}=[0, T \epsilon]^{2}$ and $\mathcal{I}_{2}=[0, T]^{2} \backslash \mathcal{I}_{1}$. Lemma 13-15 of Hu, Nualart, and Zhou 2019 imply that

$$
\begin{array}{r}
\limsup _{T \rightarrow \infty} T^{1-2 H} \int_{\mathcal{I}_{2}} e^{-u-x} \sum_{i=1}^{3}\left|\varphi_{i}\right| \mathrm{d} x \mathrm{~d} u=0 \\
\limsup _{T \rightarrow \infty} T^{1-2 H} \int_{\mathcal{I}_{1}} e^{-u-x}\left(\left|\varphi_{1}\right|+\left|\varphi_{3}\right|\right) \mathrm{d} x \mathrm{~d} u<\infty .
\end{array}
$$

Moreover, one claims that there exists a constant $c>0$ such that

$$
0<\int_{\mathcal{I}_{1}} e^{-u-x} \varphi_{2}(x) \mathrm{d} x \mathrm{~d} u=\left(1-e^{-T \epsilon}\right) \int_{0}^{T \epsilon} e^{-x} \varphi_{2}(x) \mathrm{d} x \leq \int_{0}^{T \epsilon} e^{-x} \varphi_{2}(x) \mathrm{d} x \leq c T^{2 H-1} .
$$

In fact, it is clear that there exists a constant $c_{H}>0$ such that

$$
0<(1-z)^{2 H-1}-1<c_{H} z, \quad \forall z \in\left(0, \frac{1}{2}\right] .
$$


One divides the domain of integral of $\varphi_{2}(x)$ into three parts as follows.

$$
\begin{aligned}
\int_{0}^{T \epsilon} e^{-x} \varphi_{2}(x) \mathrm{d} x & =\int_{0}^{T \epsilon} e^{-x} \mathrm{~d} x\left[\int_{x}^{2 x}+\int_{2 x}^{2 \epsilon T}+\int_{2 \epsilon T}^{T}\right]\left((t-x)^{2 H-1}-t^{2 H-1}\right)(T-t+x)^{2 H-1} \mathrm{~d} t \\
& :=J_{1}+J_{2}+J_{3}
\end{aligned}
$$

The inequality (3.12) and the monotonicity of the function $t^{2 H-1}$ imply that

$$
\begin{aligned}
& J_{1} \leq((1-\epsilon) T)^{2 H-1} \int_{0}^{T \epsilon} e^{-x} \mathrm{~d} x \frac{\left(2-2^{2 H}\right)}{2 H} x^{2 H}<c T^{2 H-1}, \\
& J_{2} \leq((1-2 \epsilon) T)^{2 H-1} \int_{0}^{T \epsilon} e^{-x} \mathrm{~d} x \int_{2 x}^{2 \epsilon T} c_{H} \frac{x}{t} t^{2 H-1} \mathrm{~d} t<c T^{2 H-1}, \\
& J_{3} \leq \int_{0}^{T \epsilon} e^{-x} x^{2 H-1} \mathrm{~d} x \int_{2 \epsilon T}^{T} c_{H} \frac{x}{t} t^{2 H-1} \mathrm{~d} t<c T^{2 H-1} .
\end{aligned}
$$

Substituting the above three inequalities into the identity (3.13), one has the inequality (3.11). Hence, one has that

$$
\limsup _{T \rightarrow \infty} T^{1-2 H} \int_{[0, T]^{2}} e^{-u-x} \sum_{i=1}^{3}\left|\varphi_{i}\right| \mathrm{d} x \mathrm{~d} u=\limsup _{T \rightarrow \infty} T^{1-2 H} \int_{\mathcal{I}_{1}} e^{-u-x} \sum_{i=1}^{3}\left|\varphi_{i}\right| \mathrm{d} x \mathrm{~d} u<\infty .
$$

Step 2.3. Speed of convergence of $\int_{[0, T]^{2}} e^{-u-x} \varphi_{4} \mathrm{~d} x \mathrm{~d} u \rightarrow \frac{1}{2} \Gamma^{2}(2 H) \sigma_{H}^{2}$. It is shown (Lemma 14 of $\mathrm{Hu}$, Nualart, and Zhou 2019) that as $T \rightarrow \infty$,

$$
\int_{[0, T]^{2}} e^{-u-x} \varphi_{4} \mathrm{~d} x \mathrm{~d} u \rightarrow \frac{1}{2} \Gamma^{2}(2 H) \sigma_{H}^{2},
$$

and the integral can be decomposed as follows:

$$
\int_{[0, T]^{2}} e^{-u-x} \varphi_{4} \mathrm{~d} x \mathrm{~d} u:=L_{1}(T)-L_{2}(T)+L_{3}(T)
$$

where

$$
\begin{aligned}
& L_{1}(T)=\int_{[0, T]^{2}} e^{-u-x} \mathrm{~d} x \mathrm{~d} u \int_{x}^{x+u} t^{2 H-1}(x+u-t)^{2 H-1} \mathrm{~d} t, \\
& L_{2}(T)=\int_{[0, T]^{2}, x<u} e^{-u-x} \mathrm{~d} x \mathrm{~d} u \int_{x}^{u}(t-x)^{2 H-1}(u-t)^{2 H-1} \mathrm{~d} t, \\
& L_{3}(T)=\int_{[0, T]^{2}} e^{-u-x} \mathrm{~d} x \mathrm{~d} u\left[\int_{x \vee u}^{T}(t-x)^{2 H-1}(t-u)^{2 H-1} \mathrm{~d} t-\int_{x+u}^{T} t^{2 H-1}(t-x-u)^{2 H-1} \mathrm{~d} t\right],
\end{aligned}
$$

Hence, one has that

$$
\limsup _{T \rightarrow \infty} T^{3-4 H}\left|\int_{[0, T]^{2}} e^{-u-x} \varphi_{4} \mathrm{~d} x \mathrm{~d} u-\frac{1}{2} \Gamma^{2}(2 H) \sigma_{H}^{2}\right|=\sum_{i=1}^{3} \limsup _{T \rightarrow \infty} T^{3-4 H}\left|L_{i}(T)-L_{i}(\infty)\right| .
$$

It is clear that

$$
\begin{aligned}
0 & <L_{1}(\infty)-L_{1}(T) \\
& =\int_{\mathbb{R}_{+}^{2} \backslash[0, T]^{2}} e^{-u-x} \mathrm{~d} x \mathrm{~d} u \int_{x}^{x+u} t^{2 H-1}(x+u-t)^{2 H-1} \mathrm{~d} t,
\end{aligned}
$$




$$
\begin{aligned}
& =\int_{\mathbb{R}_{+}^{2} \backslash[0, T]^{2}} e^{-u-x} \mathrm{~d} x \mathrm{~d} u \int_{0}^{u}(x+s)^{2 H-1}(u-s)^{2 H-1} \mathrm{~d} s, \\
& =\left[\int_{0}^{T} \mathrm{~d} x \int_{0}^{T} \mathrm{~d} s \int_{T}^{\infty} \mathrm{d} u+\int_{0}^{T} \mathrm{~d} x \int_{T}^{\infty} \mathrm{d} s \int_{s}^{\infty} \mathrm{d} u+\int_{T}^{\infty} \mathrm{d} x \int_{0}^{\infty} \mathrm{d} s \int_{s}^{\infty} \mathrm{d} u\right] \\
& \times e^{-u-x}(x+s)^{2 H-1}(u-s)^{2 H-1} \\
& :=L_{11}+L_{12}+L_{13},
\end{aligned}
$$

where

$$
\begin{aligned}
& L_{11}<\int_{0}^{T} \mathrm{~d} x \int_{0}^{T} \mathrm{~d} s \int_{T}^{\infty} e^{-u-x}(x+s)^{2 H-1}(u-T)^{2 H-1} \mathrm{~d} u<c T e^{-T}, \\
& L_{12}<\int_{0}^{T} \mathrm{~d} x \int_{T}^{\infty} \mathrm{d} s \int_{0}^{\infty} e^{-v-s-x} s^{2 H-1} v^{2 H-1} \mathrm{~d} v<c T^{2 H-1} e^{-T}, \\
& L_{13}<\int_{T}^{\infty} \mathrm{d} x \int_{0}^{\infty} \mathrm{d} s \int_{0}^{\infty} e^{-v-s-x} s^{2 H-1} v^{2 H-1} \mathrm{~d} v<c e^{-T},
\end{aligned}
$$

which imply that $L_{1}(T) \rightarrow L_{1}(\infty)$ with an exponential rate as $T \rightarrow \infty$. It is obvious that $L_{2}(T) \rightarrow L_{2}(\infty)$ also with an exponential rate as $T \rightarrow \infty$. In fact,

$$
\begin{aligned}
0 & <L_{2}(\infty)-L_{2}(T) \\
& =\int_{\mathbb{R}_{+}^{2} \backslash[0, T]^{2}, x<u} e^{-u-x} \mathrm{~d} x \mathrm{~d} u \int_{x}^{u}(t-x)^{2 H-1}(u-t)^{2 H-1} \mathrm{~d} t \\
& =B(2 H, 2 H) \int_{T}^{\infty} \mathrm{d} x \int_{x}^{\infty} e^{-u-x}(u-x)^{4 H-1} \mathrm{~d} u \\
& =B(2 H, 2 H) \Gamma(2 H) \int_{T}^{\infty} e^{-2 x} \mathrm{~d} x<c e^{-2 T} .
\end{aligned}
$$

Since $(t-x)(t-u) \geq t(t-x-u)$ for $x, u>0$, the symmetry and the monotonicity of the function $t^{2 H-1}$ imply that

$$
\begin{aligned}
& \frac{1}{2}\left|L_{3}(\infty)-L_{3}(T)\right| \\
= & \mid \int_{0<u<x<t, t \leq x+u, t>T} e^{-u-x}(t-x)^{2 H-1}(t-u)^{2 H-1} \mathrm{~d} t \mathrm{~d} x \mathrm{~d} u \\
- & \left(-\int_{0<u<x<t, t>x+u, t>T} e^{-u-x}(t-x)^{2 H-1}(t-u)^{2 H-1} \mathrm{~d} t \mathrm{~d} x \mathrm{~d} u\right. \\
& \left.+\int_{0<u<x, t>x+u, t>T} e^{-u-x} t^{2 H-1}(t-x-u)^{2 H-1} \mathrm{~d} t \mathrm{~d} x \mathrm{~d} u\right) \mid \\
:= & \left|K_{1}(T)-K_{2}(T)\right|<K_{1}(T)+K_{2}(T) .
\end{aligned}
$$

It is clear that

$$
\begin{aligned}
K_{1}(T) & =\int_{0<u<x<t \leq x+u, t>T} e^{-u-x}(t-x)^{2 H-1}(t-u)^{2 H-1} \mathrm{~d} t \mathrm{~d} x \mathrm{~d} u \\
& =\int_{T}^{\infty} \mathrm{d} t \int_{\frac{t}{2}}^{t} \mathrm{~d} x \int_{t-x}^{x} \mathrm{~d} u e^{-u-x}(t-x)^{2 H-1}(t-u)^{2 H-1}
\end{aligned}
$$




$$
\begin{aligned}
& <\int_{T}^{\infty} e^{-t} \mathrm{~d} t \int_{\frac{t}{2}}^{t}(t-x)^{2 H-1} \mathrm{~d} x \int_{t-x}^{x}(t-u)^{2 H-1} \mathrm{~d} u \\
& <c T^{4 H} e^{-T}
\end{aligned}
$$

and

$$
\begin{aligned}
K_{2}(T) & =\int_{T}^{\infty} \mathrm{d} t \int_{0}^{\frac{t}{2}} \mathrm{~d} u \int_{u}^{t-u} \mathrm{~d} x e^{-u-x}\left[t^{2 H-1}(t-x-u)^{2 H-1}-(t-x)^{2 H-1}(t-u)^{2 H-1}\right] \\
& =\int_{T}^{\infty} e^{-t} t^{2 H-1} \mathrm{~d} t \int_{0}^{\frac{t}{2}} \mathrm{~d} u \int_{0}^{t-2 u} e^{z} z^{2 H-1} \mathrm{~d} z-\int_{T}^{\infty} e^{-2 t} \mathrm{~d} t \int_{\frac{t}{2}}^{t} e^{y} y^{2 H-1} \mathrm{~d} y \int_{t-y}^{y} e^{z} z^{2 H-1} \mathrm{~d} z,
\end{aligned}
$$

where the last equality is by the change of variables $t-x-u=z$ and $t-x=z, t-u=y$ respectively. Then the L'Hospital's rule implies that

$$
\begin{aligned}
& \lim _{T \rightarrow \infty} \frac{2(3-4 H)}{T^{4 H-3}} K_{2}(T) \\
& =\lim _{T \rightarrow \infty} \frac{2}{e^{2 T} T^{4 H-4}}\left[e^{T} T^{2 H-1} \int_{0}^{\frac{T}{2}} \mathrm{~d} u \int_{0}^{T-2 u} e^{z} z^{2 H-1} \mathrm{~d} z-\int_{\frac{T}{2}}^{T} e^{y} y^{2 H-1} \mathrm{~d} y \int_{T-y}^{y} e^{z} z^{2 H-1} \mathrm{~d} z\right] \\
& =\lim _{T \rightarrow \infty} \frac{e^{T} T^{2 H-1}}{e^{2 T} T^{4 H-4}}\left[\left(1+\frac{2 H-1}{T}\right) \int_{0}^{\frac{T}{2}} \mathrm{~d} u \int_{0}^{T-2 u} e^{z} z^{2 H-1} \mathrm{~d} z-\frac{1}{2} \int_{0}^{T} e^{z} z^{2 H-1} \mathrm{~d} z+\frac{1}{2} B(2 H, 2 H) T^{2 H}\right] \\
& =\lim _{T \rightarrow \infty} \frac{1}{e^{T} T^{2 H-2}}\left[(T+2 H-1) \int_{0}^{\frac{T}{2}} \mathrm{~d} u \int_{0}^{T-2 u} e^{z} z^{2 H-1} \mathrm{~d} z-\frac{T}{2} \int_{0}^{T} e^{z} z^{2 H-1} \mathrm{~d} z\right] \\
& =\lim _{T \rightarrow \infty} \frac{1}{e^{T} T^{2 H-2}}\left[\int_{0}^{T} e^{z} z^{2 H-1} \mathrm{~d} z-e^{T} T^{2 H-1}\right] \\
& =(1-2 H) .
\end{aligned}
$$

Hence it follows from (3.16) that one has that

$$
\limsup _{T \rightarrow \infty} T^{3-4 H}\left|\int_{[0, T]^{2}} e^{-u-x} \varphi_{4} \mathrm{~d} x \mathrm{~d} u-\frac{1}{2} \Gamma^{2}(2 H) \sigma_{H}^{2}\right| \leq \frac{1-2 H}{3-4 H}<\infty .
$$

Combining (3.17) and (3.14) with (3.10), one has that

$$
\begin{aligned}
& \limsup _{T \rightarrow \infty} T^{1-2 H}\left|I_{11}(T)-\frac{1}{2} \Gamma^{2}(2 H) \sigma_{H}^{2}\right| \\
& \leq \limsup _{T \rightarrow \infty} T^{1-2 H} \int_{[0, T]^{2}} e^{-u-x} \sum_{i=1}^{3}\left|\varphi_{i}\right| \mathrm{d} x \mathrm{~d} u+\limsup _{T \rightarrow \infty} T^{1-2 H}\left|\int_{[0, T]^{2}} e^{-u-x} \varphi_{4} \mathrm{~d} x \mathrm{~d} u-\frac{1}{2} \Gamma^{2}(2 H) \sigma_{H}^{2}\right| \\
& =\limsup _{T \rightarrow \infty} T^{1-2 H} \int_{[0, T]^{2}} e^{-u-x} \sum_{i=1}^{3}\left|\varphi_{i}\right| \mathrm{d} x \mathrm{~d} u<\infty
\end{aligned}
$$

Step 2.4. Speed of convergence $I_{12}(T) \rightarrow \frac{1}{2} \Gamma^{2}(2 H) \sigma_{H}^{2}$. It follows from (6.36) of Hu, Nualart, and Zhou 2019 that

$$
I_{12}(T)=\int_{[0, T]^{2}} e^{-u-x}\left(\varphi_{4}+\varphi_{5}\right) \mathrm{d} x \mathrm{~d} u
$$


where

$\varphi_{5}=\int_{x}^{T}\left(\operatorname{sgn}(x+u-t)|x+u-t|^{2 H-1}(T-t)^{2 H-1}-\operatorname{sgn}(u-t)|u-t|^{2 H-1}(T-t+x)^{2 H-1}\right) \mathrm{d} t$.

Similar to Step 2.2, it follows from Lemma 13-15 of Hu, Nualart, and Zhou 2019 that as $T \rightarrow \infty$,

$$
\limsup _{T \rightarrow \infty} T^{1-2 H} \int_{[0, T]^{2}} e^{-u-x}\left|\varphi_{5}\right| \mathrm{d} x \mathrm{~d} u=\limsup _{T \rightarrow \infty} \int_{\mathcal{I}_{1}} e^{-u-x}\left|\varphi_{5}\right| \mathrm{d} x \mathrm{~d} u<\infty .
$$

Combining (3.17) and (3.20) with (3.19), one has that

$$
\limsup _{T \rightarrow \infty} T^{1-2 H}\left|I_{12}(T)-\frac{1}{2} \Gamma^{2}(2 H) \sigma_{H}^{2}\right| \leq \limsup _{T \rightarrow \infty} T^{1-2 H} \int_{[0, T]^{2}} e^{-u-x}\left|\varphi_{5}\right| \mathrm{d} x \mathrm{~d} u<\infty .
$$

Combining (3.18) and (3.21) with (3.8), one has that

$$
\begin{aligned}
& \limsup _{T \rightarrow \infty} T^{1-2 H}\left|I_{1}(T)-(H \Gamma(2 H))^{2}\right| \\
& \leq \limsup _{T \rightarrow \infty} T^{1-2 H}\left|I_{11}(T)-\frac{1}{2} \Gamma^{2}(2 H) \sigma_{H}^{2}\right|+\limsup _{T \rightarrow \infty} T^{1-2 H}\left|I_{12}(T)-\frac{1}{2} \Gamma^{2}(2 H) \sigma_{H}^{2}\right| \\
& <\infty .
\end{aligned}
$$

That is to say, the speed of convergence $I_{1} \rightarrow[H \Gamma(2 H)]^{2}$ is at least $T^{2 H-1}$.

Step 3. Speeds of convergences $I_{2}(T), I_{3}(T) \rightarrow 0$. One has that

$$
\begin{aligned}
& \frac{2 \sigma_{H}^{2}}{H^{2}} I_{2}(T) \\
= & \frac{1}{T e^{T}} \int_{[0, T)^{3}} e^{t_{2}-\left|t_{1}-s\right|} \operatorname{sgn}\left(s-t_{1}\right)\left(s^{2 H-1}+(T-s)^{2 H-1}\right)\left(t_{2}^{2 H-1}-\operatorname{sgn}\left(t_{2}-t_{1}\right)\left|t_{2}-t_{1}\right|^{2 H-1}\right) \mathrm{d} s \mathrm{~d} \vec{t} .
\end{aligned}
$$

Comparing it with the identity (3.9), one has that

$$
\frac{2 \sigma_{H}^{2}}{H^{2}} I_{2}(T)=\frac{1}{T} I_{12}(T) .
$$

Hence, the equalities (3.15) and (3.19) imply that there exists a constant $c>0$ such that for $T$ large enough,

$$
\left|I_{2}(T)\right| \leq \frac{c}{T}
$$

By the symmetry, one has that $I_{2}=I_{3}$.

Step 4. Speed of convergence $I_{4}(T) \rightarrow 0$. It is clear that there exists a constant $c>0$ such that as $T$ large enough,

$$
I_{4}(T)=\frac{H^{2}}{2 \sigma_{H}^{2} T e^{2 T}}\left[\int_{0}^{T} e^{t}\left(t^{2 H-1}+(T-t)^{2 H-1}\right) \mathrm{d} t\right]^{2}<\frac{c}{T} .
$$

Finally, substituting speeds of convergences obtained at Step 2-4 to (3.7), one has the desired conclusion.

After the above three lemmas are shown, proof of Theorem 1.1 is almost the same as that of the case of $H \in\left[\frac{1}{2}, \frac{3}{4}\right)$, please refer to Chen, Kuang, and Li 2018. But for the reader's convenience, one still writes it here. The only difference is the upper bound in the inequality (3.22) given below. 
Proof of Theorem 1.1. It follows from Theorem 2.4, Lemma 3.2 and Eq.(2.10)-(2.14) that there exists a constant $C_{\theta, H}$ such that for $T$ large enough,

$$
\begin{aligned}
& \sup _{z \in \mathbb{R}}\left|P\left(\sqrt{\frac{T}{\theta \sigma_{H}^{2}}}\left(\hat{\theta}_{T}-\theta\right) \leq z\right)-P(Z \leq z)\right| \leq \\
& C_{\theta, H} \times \max \left\{\left|b_{T}^{2}-2\left\|f_{T}\right\|^{2}\right|,\left\|f_{T} \otimes_{1} f_{T}\right\|,\left\|f_{T} \otimes_{1} g_{T}\right\|,\left|\left\langle f_{T}, g_{T}\right\rangle\right|,\left\|g_{T}\right\|^{2},\left\|g_{T} \otimes_{1} g_{T}\right\|\right\} .
\end{aligned}
$$

Denote $a=H \Gamma(2 H) \theta^{-2 H}$. Lemma 3.2 and Lemma 3.6 imply that there exists a constant $c$ such that for $T$ large enough,

$$
\left|b_{T}^{2}-2\left\|f_{T}\right\|^{2}\right| \leq\left|b_{T}^{2}-a^{2}\right|+\left|2\left\|f_{T}\right\|^{2}-a^{2}\right| \leq c \times \frac{1}{T^{1-2 H}} .
$$

Corollary 3.5 implies that there exists a constant $c$ such that for $T$ large enough,

$$
\left\|f_{T} \otimes_{1} g_{T}\right\|,\left|\left\langle f_{T}, g_{T}\right\rangle\right|,\left\|g_{T} \otimes_{1} g_{T}\right\| \leq c \times \frac{1}{\sqrt{T}}, \quad\left\|g_{T}\right\|^{2} \leq c \times \frac{1}{T} .
$$

Combining (3.1) with the above inequalities, one obtains that (1.3) holds.

Acknowledgements: We would like to gratefully thank the referee for very valuable suggestions which lead to the improvement of the new version. Y. Chen is supported by NSFC (No.11871079).

\section{REFERENCES}

[1] Barboza, L. A., and F. G. Viens. 2017. Parameter estimation of Gaussian stationary processes using the generalized method of moments. Electronic Journal of Statistics 11: 401-439.

[2] Bercu, B., L. Coutin, and N. Savy. 2011. Sharp large deviations for the fractional Ornstein-Uhlenbeck process. Theory Probab Appl 55(4): 575-610.

[3] Bishwal, J. 2000. Sharp Berry-Esséen bound for the maximum likelihood estimators in the OrnsteinUhlenbeck process. Sankhya Series A 62: 1-10.

[4] Bishwal, J. 2008. Parameter Estimation in Stochastic Differential Equations, Vol.1923 of Lecture Notes in Mathematics, Berlin: Springer.

[5] Brouste, A. and M. Kleptsyna. 2010. Asymptotic properties of MLE for partially observed fractional diffusion system. Stat Inference Stoch Process 13(1):1-13.

[6] Chen, Y., N.-H. Kuang, and Y. Li. 2018. Berry-Esséen bound for the Parameter Estimation of Fractional Ornstein-Uhlenbeck Processes, https://arxiv.org/abs/1806.01487.

[7] Cheridito P., and D. Nualart. 2005. Stochastic integral of divergence type with respect to fractional Brownian motion with Hurst parameter $H \in\left(0, \frac{1}{2}\right)$. Ann Inst $H$ Poincare 41:1049-1081.

[8] Es-Sebaiy, K. 2013. Berry-Esséen bounds for the least squares estimator for discretely observed fractional Ornstein-Uhlenbeck processes. Statistics \& Probability Letters 83(10): 2372-2385.

[9] Es-Sebaiy, K., Viens, F. 2018 Optimal rates for parameter estimation of stationary Gaussian processes. Stochastic Processes and their Applications, https://doi.org/10.1016/j.spa.2018.08.010.

[10] Hu, Y., M. Jolis, and S. Tindel. 2013. On Stratonovich and Skorohod stochastic calculus for Gaussian processes. Ann Probab 41(3A):1656-1693.

[11] Hu, Y., and D. Nualart. 2010. Parameter estimation for fractional Ornstein-Uhlenbeck processes. Statistics Probability Letters 80(11-12):1030-1038.

[12] Hu, Y., D. Nualart, and H. Zhou. 2019. Parameter estimation for fractional Ornstein-Uhlenbeck processes of general Hurst parameter. Stat Inference Stoch Process 22: 111-142.

[13] Jolis, M. 2007. On the Wiener integral with respect to the fractional Brownian motion on an interval. Journal of Mathematical Analysis and Applications 330:1115-1127. 
[14] Kim, Y. T., and H. S. Park. 2017a. Optimal Berry-Esséen bound for an estimator of parameter in the Ornstein-Uhlenbeck process. Journal of the Korean Statistical Society 46(3): 413-425.

[15] Kim, Y. T., and H. S. Park. 2017b. Optimal Berry-Esséen bound for statistical estimations and its application to SPDE. Journal of Multivariate Analysis 155: 284-304.

[16] Kleptsyna, M.L., and A. Le Breton. 2002. Statistical analysis of the fractional Ornstein-Uhlenbeck type process. Stat Inference Stoch Process 5: 229-248.

[17] Onsy, B.E., K. Es-Sebaiy, and F.G. Viens. 2017. Parameter Estimation for a partially observed OrnsteinUhlenbeck process with long-memory noise. Stochastics An International Journal of Probability and Stochastic Processes 89(2): 431-468.

[18] Pipiras, V., and M.S. Taqqu. 2000. Integration questions related to fractional Brownian motion. Probab Theory Relat Fields 118: 251-291.

[19] Pipiras, V., and M.S. Taqqu. 2001. Are classes of deterministic integrands for fractional Brownian motion on an interval complete? Bernoulli 6 873-897

[20] Sottinen, T., and L. Viitasaari. 2018. Parameter estimation for the langevin equation with stationaryincrement gaussian noise. Stat Inference Stoch Process 21(3): 569-601.

[21] Tao, T. 2011. An introduction to measure theory. Vol. 126 of Graduate Studies in Mathematics. Providence: American Mathematical Society.

[22] Taylor, A. E. 1952. L'Hospital's rule, Amer. Math. Monthly 59: 20-24.

[23] Tudor, C. and F. Viens. 2007. Statistical aspects of the fractional stochastic calculus. Ann Statist 35(3): $1183-1212$

College of Mathematics and Information Science, Jiangxi Normal University, Nanchang, 330022, JiANGXI, ChINA

E-mail address: zhishi@pku.org.cn

School of Mathematics and Computional Science, Xiangtan University, Xiangtan, 411105, HuNAN, ChINA

E-mail address: liying@xtu.edu.cn 